\title{
Dynamic Electromagnetic Field Measurements of Clustered Hall Thrusters
}

\author{
Robert B. Lobbia* and Alec D. Gallimore. ${ }^{\dagger}$ \\ The University of Michigan, Ann Arbor, MI, 48109
}

\begin{abstract}
In an effort to verify the existence of and study the characteristics of the dynamic magnetic fields generated by supplying inner and outer coil Hall thruster electromagnets with wide bandwidth (DC to $200 \mathrm{kHz}$ ) currents, B-dot probe experiments have been performed. Several different locations were mapped and all three components of the magnetic field were recorded. A Helmholtz coil is used for high frequency calibration of the B-dot probe while a commercial Gaussmeter is used for DC magnetic field measurements and calibrations. These experiments show the electromagnets' coils do indeed experience inductive behavior at the higher frequencies but that the magnetic performance agrees well with theory. Voltage signals from the various current and magnetic field probes are processed into frequency space to visualize signal power spectrums. Several Bode plots are also constructed using the excitation current as the input of transfer functions representing the magnetic field frequency response to chirp-type excitation.
\end{abstract}

\section{Introduction}

Since their inception decades ago, closed drift Hall Thrusters have predominantly been operated with static electric and magnetic fields. Popular among spacecraft designers and mission planners for their mass savings (i.e. enormous specific impulse ratings as high as $2000 \mathrm{sec}$ ) and grid-less construction, Hall thrusters are becoming increasingly important in the exploration of the solar system and the future of telecommunication satellites. Operating at voltages less than 600 Volts requires a smaller and simpler PPU (power processing unit) than many other types of electric propulsion systems and therefore Hall thrusters easily integrate with telecommunication satellite buses [1]. Larger thrust requirements for many missions led to the concept of clustering [2] medium power Hall thrusters which are more easily developed than a single $100 \mathrm{~kW}+$ high power Hall thruster. Clustering also adds a level of redundancy for each thruster, provided each uses its own PPU and propellant delivery system. An array of clustered thrusters could also employ thrust vectoring for trajectory maneuvers as well as a set of $\mathrm{N}$ fully optimized thrust set points with the operation of 1 to $\mathrm{N}$ thrusters.

\section{A. Plasma Instabilities and Oscillatory Modes}

The electromagnetic nature of plasma gives rise to a very broad spectrum of observable instabilities; in the interest of electric propulsion this range is around $1 \mathrm{kHz}$ to $2 \mathrm{GHz}[3,4]$. The bulk of prior electric propulsion research on plasma instabilities has focused on characterizing the magnitude and frequency characteristics [5-7] and suggesting theories of the physics creating the oscillations $[4,8,9]$. Virtually no research has attempted actively controlling and minimizing the oscillatory modes [10] so commonly observed in Hall thrusters and other electric propulsion devices. While the gigahertz modes are clearly too rapid to attempt to control in closed loop feedback, there exist several potentially controllable lower frequency modes: rotating spike $(\sim 50 \mathrm{kHz})$, breathing mode $(20-$ $50 \mathrm{kHz})$, and other ionization instabilities $(<100 \mathrm{kHz})$.

Fusion research has shown controllability of some of the instabilities in plasmas by the use of electromagnetic coils and wall electrode panels [11, 12]. The high frequencies of plasma instabilities requires the use of electromagnetic actuators; and this paper studies the feasibility of using the inner electromagnetic coil of a Hall thruster in the feedback control observed of instabilities.

\footnotetext{
*PhD Candidate, Aerospace Engineering Dept., FXB Building Room 1056, AIAA Student Member.

${ }^{\dagger}$ Arthur F. Thurnau Professor, Aerospace Engineering Dept., Associate Fellow AIAA.
} 


\section{Motivation}

The various instabilities observed in electric propulsion devices are primarily detrimental [4] since rapid current oscillations can damage the PPU and cause additional sputtering and erosion. The coupling of the ionization process to these instabilities $[4,8]$ strongly suggests that efficiency of a Hall thruster can be altered if the instabilities can be mitigated. Thus, a high-speed feedback control system has been developed and in this paper the use of

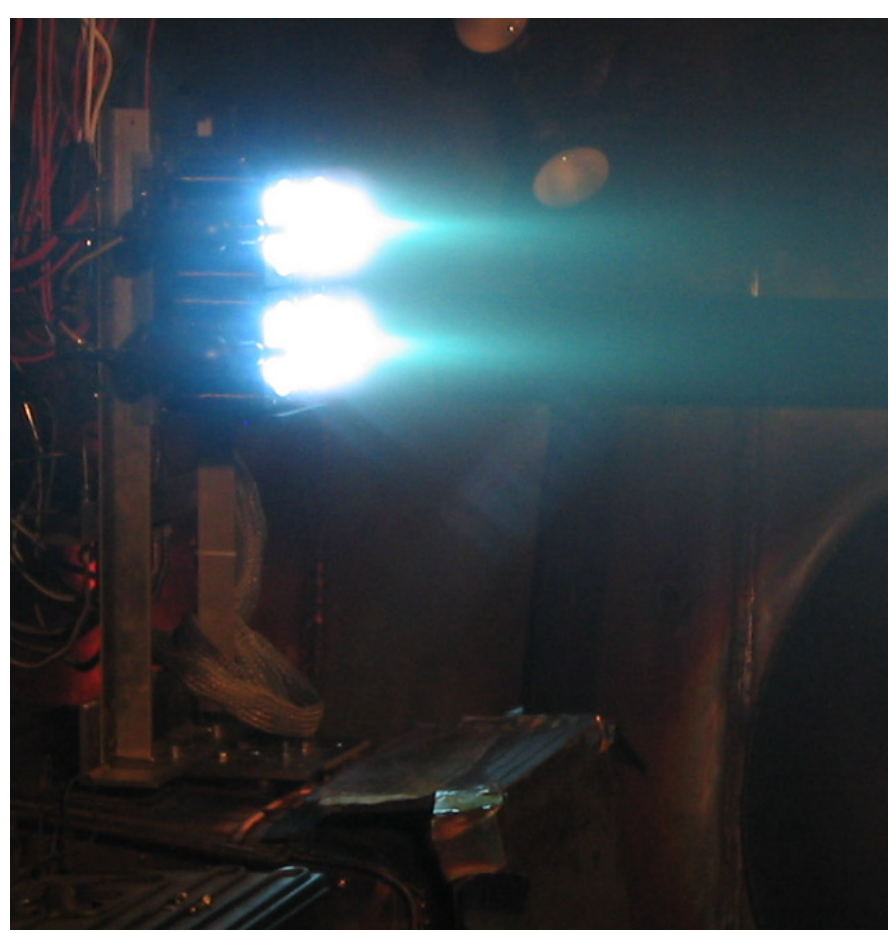

Figure 1. BHT-600 Cluster (June 2006): Xenon discharge of $300 \mathrm{~V}$ and $2 \mathrm{~A}$ per thruster in PEPL's LVTF (Large Vacuum Test Facility) mounted on thrust stand for performance measurements. electromagnetic actuation is studied in detail. In addition to controlling observed Hall thruster instabilities, another objective of the controller development is the minimization of clustering effects such as discharge current fluctuation crosstalk between thrusters [2]. Ultimately, measurements of thrust and erosion will show the effectiveness of controlling plasma instabilities. It seems reasonable to believe that a reduction in the amplitude of discharge current must fundamentally lead to a more efficient transfer of energy to the ion particles and thus higher thrust with less erosion (from lower $\mathrm{Xe}^{++}$generation and better focusing).

\section{Experimental Setup}

\section{B. Atmospheric Electromagnetic Field Measurements}

All the experimental results presented have been obtained under normal atmospheric conditions. The plasma discharge of a Hall thruster does certainly affect the magnetic field [13] but such effects are neglected here to as to focus purely on the electromagnetic actuation scheme. The Hall thrusters used in these experiments are Busek BHT-600 thrusters setup in a 2 by 2 cluster. Each thruster has its own independent set of power supplies and propellant lines. The lack of a plasma discharge simplifies the experiment and only the inner electromagnet and outer electromagnet power supplies are operated for each thruster.

\section{Magnetic Probes}

Two types of magnetic probes are used. A LakeShore Model 460 3-Axis Gaussmeter is used for measure DC and low frequency magnetic fields (less than $400 \mathrm{~Hz}$ ). Additionally, two inductive loop type "B-dot" probes [13] were built and calibrated for use in measuring dynamic magnetic fields $(100 \mathrm{~Hz}-100 \mathrm{kHz})$. Two Helmholtz coils were constructed with known geometries in order to calibrate the B-dot probes. The first Helmholtz coil eventually shorted out (enamel coating of copper magnet wire melted) and thus a second coil was constructed with a higher Qfactor (quality factor) using larger gauge (20AWG) magnet wire to minimize heating effects. The mean coil radius in the second Helmholtz coil was $1.23 \mathrm{~cm}$ which is also the axial separation of coils (following typical Helmholtz coil geometry). The formula for the magnetic field inside of the Helmholtz coils is then [14]:

$$
\left.B_{\text {Helmholtz }}\right|_{\text {center }}(\text { Gauss })=0.4032 \frac{N I}{R} \quad \text { Eqn.1 }
$$

Where $N$ is the number of turns is each coil ( $\mathrm{N}=11$ here), $I$ is the current flowing through each coil in Amps, and $\mathrm{R}$ is the mean coil radius in centimeters. A B-dot probe is quite similar to a set of Helmholtz coils except that there is only a single coil. While two probes were built, one was damaged after initial testing: 

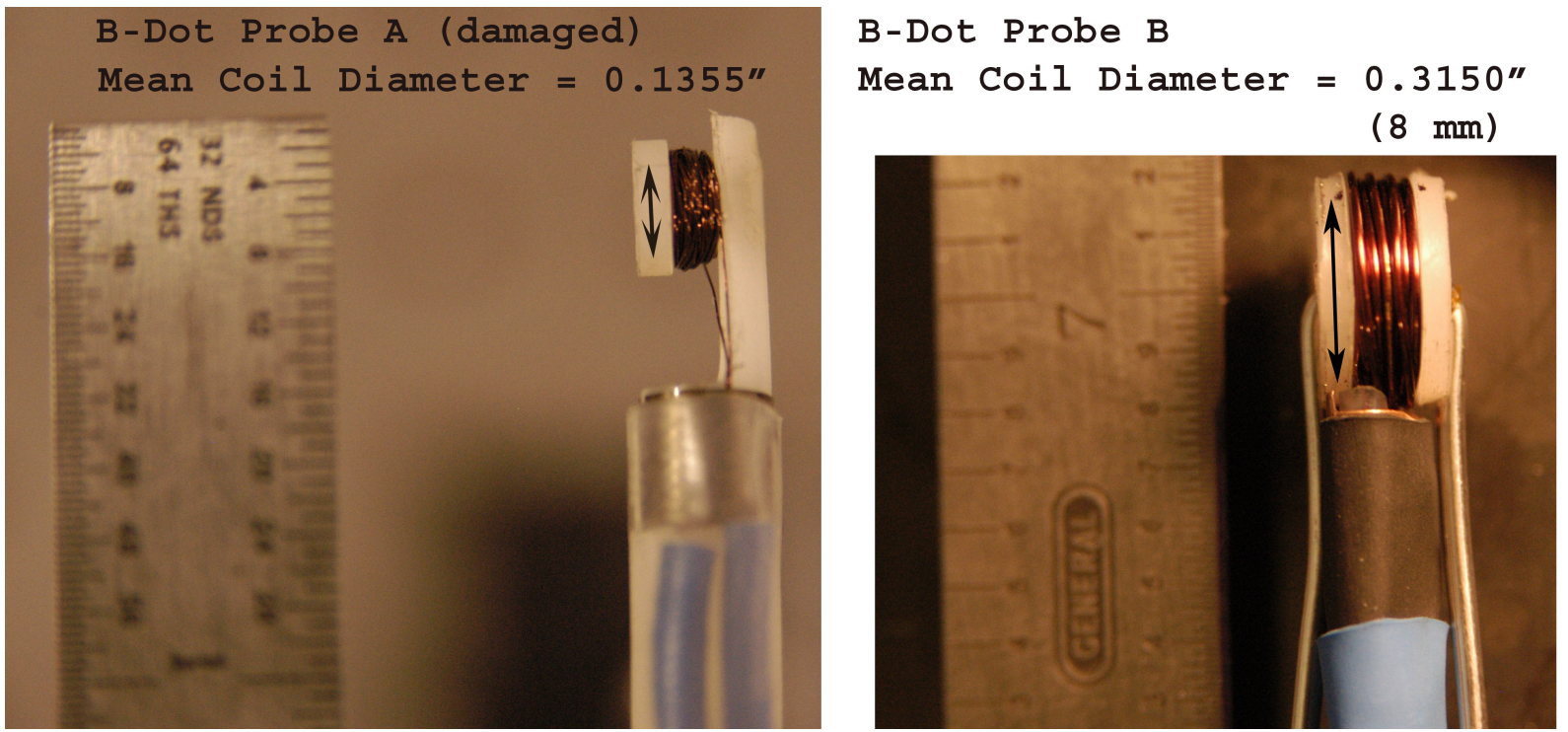

Figure 2. B-Dot Probes. Close-up photos of the two B-dot probes. While both probes were successfully used and calibrated, the small gauge wire of probe A proved too fragile and was damaged so that most data was then collected with probe B.

The second probe has a mean coil diameter of $8 \mathrm{~mm}$ with 25 turns of 26AWG copper wire (see figure 2). Faraday's Law is then used to relate the measured voltage signal induced in the B-dot probes due to the changing magnetic flux setup in a dynamic magnetic field:

$$
V_{B-d o t}=-N_{\text {probe }} A_{\text {probe }} \frac{d B}{d t} \quad \text { Eqn.2 }
$$

Here, $N_{\text {probe }}=25$, is the turns of wire in the B-dot probe and $A_{\text {probe }}$ is the area of the probe loop with all units in Equation 2 in SI. For the experiments performed, the $\frac{d B}{d t}$ term is quite small and it is necessary to amplify the induced voltage signal. An OP37 op-amp is used to amplify the signal prior to sampling via the data acquisition system. A $500 \mathrm{kHz}$ low pass filter also precedes the DAQ system to prevent aliasing and high frequency pickup (since the B-dot probe's sensitivity increases with increasing frequency).

\section{Data Acquisition}

For the fixed frequency and DC measurements a digital 4-channel Tektronix 3-GHz Oscilloscope was utilized. For the higher frequency datasets a 500kHz 8-channel PCI-based AtoD card was used. A sample and hold buffering scheme allowed for true simultaneous channel sampling thereby ensuring accurate phase calculations between the signals. For all the non-DC experiments a Wavetek function generator is used to create a sinusoidal input to the power supply driving the electromagnet whose varying magnetic fields are being evaluated. A TTL output from the function generator is used to trigger the oscilloscope so that 512-windowed averaging can be performed on the signals. 


\section{E. Current Measurements}

A total of five different types of current measurements are used to ensure an accurate current measurement at high and low frequencies:

$$
\begin{array}{ll}
\mathrm{I}_{\mathrm{NT}-5} \text {, Magneto Resistance Sensor } & 100 \mathrm{kHz} \text { Freq. Response } \\
\mathrm{I}_{\text {tektronix }}, \text { Hall Probe Sensor } & 5 \mathrm{MHz} \text { Freq. Response } \\
\mathrm{I}_{\text {shunt,direct }} \text { Shunt Measurement } & \text { Infinite Freq. Response } \\
\mathrm{I}_{\text {shunt,amplified }}, \text { Shunt Measurement (Amplified) } & \text { 200kHz Freq. Response } \\
\mathrm{I}_{\text {mon }} \text {, Power Supply Current Monitor } & \text { Unknown Freq. Response }
\end{array}
$$

Table 1. Current Sensor Frequency Responses

To the right of each current sensor is listed the rated frequency response of the sensor. Each sensor has different advantages, notably the first two sensors provide complete voltage isolation between the DAQ system and the electromagnetic power lines. The hall probe sensor has a huge bandwidth but it is also noisy and a low (50mV) level signal whereas the magneto resistive probe has a balanced bridge amplification circuit that lowers the frequency response but outputs a higher ( $1 \mathrm{~V})$ level signal. The shunt signal is also a low level signal $(50 \mathrm{mV})$ and it is not isolated, requiring a direct connection of the DAQ to the electromagnetic line. The second shunt signal is isolated and then amplified. Finally, the KEPCO power supply analog current monitor output is measured and compared to the other signals since the manufacturer's specifications are unclear about the frequency response of this signal.

\section{Experimental Results}

\section{F. Helmholtz Coil Results}

Before measuring dynamic magnetic fields on the BHT600 Hall thruster, a set of Helmholtz coils was used to calibrate the B-dot probe. The known geometry of Helmholtz coils allows for us to estimate the theoretical internal magnetic field. As a check of this field we inserted the LakeShore Gaussmeter to measure the DC magnetic field (results shown in figure $3)$.

Agreement between the measured and the theoretical magnetic fields is within about $4 \%$. We use this data to compute a calibration factor for our Helmholtz coil thereby correcting our theoretical value. Next, a series of fixed

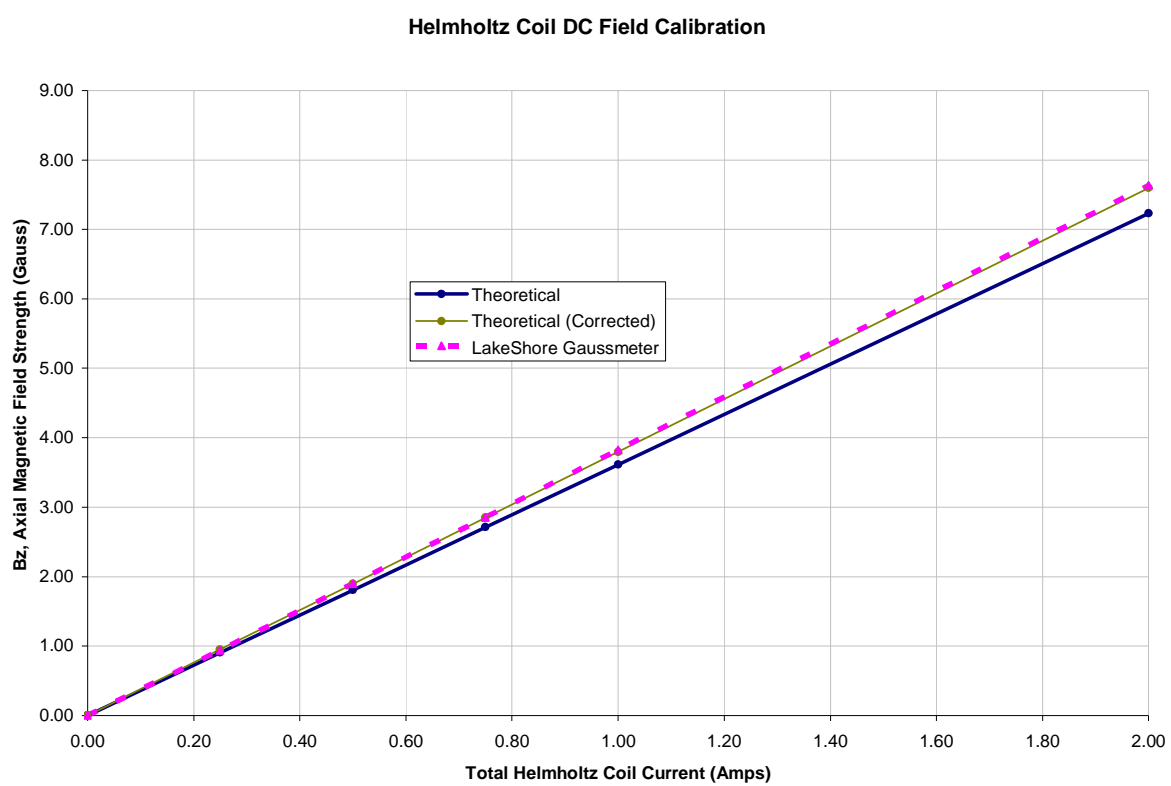

Figure 3. DC Helmholtz Coil Calibration. 
frequency experiments were carried out to calibrate our B-dot probe at frequencies between $100 \mathrm{~Hz}$ and $100 \mathrm{kHz}$ :

Helmholtz Coil Low and High Frequency Operation

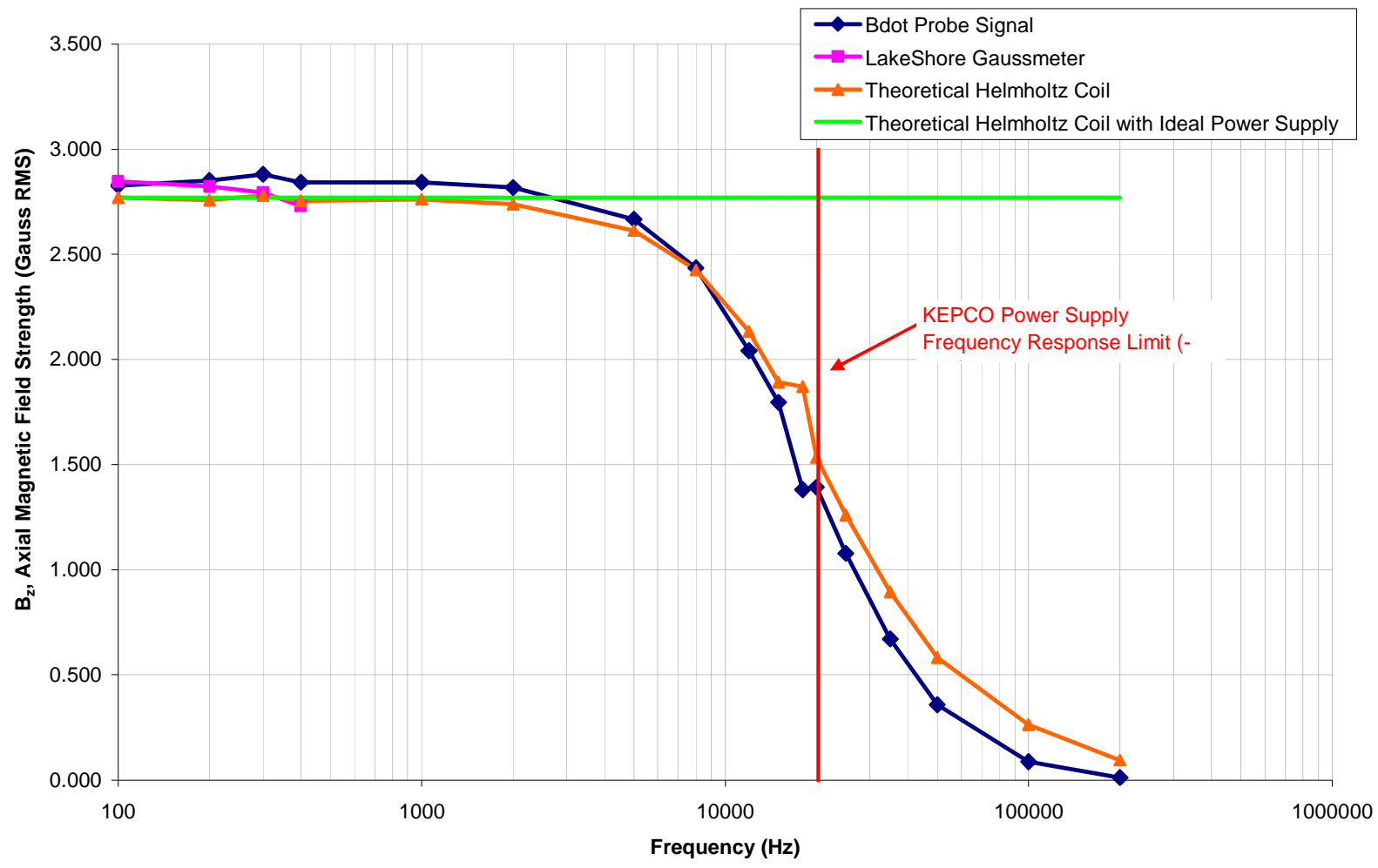

Figure 4. B-dot Probe Frequency Calibration.

Here we again see excellent agreement between the expected field and the measured field. Since the B-dot probe is actually measuring $\frac{d B}{d t}$ we have integrated to compute the RMS value of the magnetic field:

$$
B_{r m s}(\text { Tesla })=\frac{V_{B-d o t, r m s}}{2 \pi \eta G N_{\text {probe }} A_{\text {probe }} f} \quad \text { Eqn.3 }
$$

The $2 \pi f=\omega$ term in the denominator simply arises from integration of a sine wave at frequency $f$, in Hertz. $G$ in this equation is the gain set by the op-amp and for most experiments a value of 11005 is used. A B-dot probe efficiency factor, $\eta$, was introduced here but the satisfactory agreement observed in figure 4 prompts us to set this to one. The integration also introduces a $90^{\circ}$ phase shift between the magnetic field and the voltage as shall be seen more clearly later on. The drop-off that occurs as the frequency increases beyond $20 \mathrm{kHz}$ occurs due to limitations in the power supply's frequency response. An ideal power supply with an infinite frequency response would not exhibit this trend as is indicated in figure 4. The LakeShore Gaussmeter was configured to measure RMS values and those results are also shown, but the extremely limited frequency response of that probe proves of minimal usefulness at the frequencies of interest here. Besides providing a solid DC reference field measurement, the other useful aspect of the LakeShore Gaussmeter probe was in examining the uniformity of the magnetic field interior to the Helmholtz coils. As the coils were only 3 times the size of the B-dot probe utilized, there was some concern about field non-uniformity that may adversely effect the calibration. Thanks to the clever design of the Helmholtz coil pair (which is designed to create an optimally uniform magnetic field) the DC- $400 \mathrm{~Hz}$ magnetic fields measured 
over the bulk of the internal volume varied by merely $+/-0.01$ gauss (or on the order of $<1 \%$ of the total magnetic field)!

\section{G. Chirp Experiments in the Time Domain}

Measuring the response to fixed frequency sine waves provides incredibly clean signals with precise voltage RMS values due to the trigging/averaging scheme used on the scope (about 5 million voltage points are used to make a single RMS reading!) and the high voltage resolution of the scope. But such measurements are tedious and can verily miss key features of interest in the response. Another approach is to use a so-called "chirp" waveform. Mathematically, a chirp is still a sine wave but with a time dependant frequency:

$$
\begin{aligned}
& y_{\text {chirp }}(t)=A \cdot \sin (2 \pi \cdot f(t) \cdot t) \\
& f(t)=f_{\text {start }}+\frac{\left(f_{\text {stop }}-f_{\text {start }}\right)}{t_{\text {sweep }}} \cdot t \quad \text { Eqn.4 }
\end{aligned}
$$

In using signals of this form to excite the electromagnetic field, a single chirp waveform spanning the time, $t_{\text {sweep }}$, can be used to analyze the entire frequency response between the frequencies $f_{\text {start }}$ and $f_{\text {stop }}$. For typical chrip experiments $f_{\text {start }}=200 \mathrm{~Hz}, f_{\text {stop }}=100 \mathrm{kHz}$, and $t_{\text {sweep }}=10$-seconds; thus, the sine wave frequency increases about $10 \mathrm{kHz}$ each second. If our electromagnet was instead a loudspeaker, the noise would actually sound like a "chirping" bird, hence the name. A PC-based DAQ system then samples the chirp signal and the electromagnetic response signals from the various probes. In a two signal input-output experiment each signal is simultaneously acquired at $250 \mathrm{kHz}$ for about 20 seconds (a $125 \mathrm{kHz}$ Nyquist frequency) which is sufficient for the measurement of the $200 \mathrm{~Hz}-100 \mathrm{kHz}$ chirp bandwidth. A small portion of such a signal is shown below:

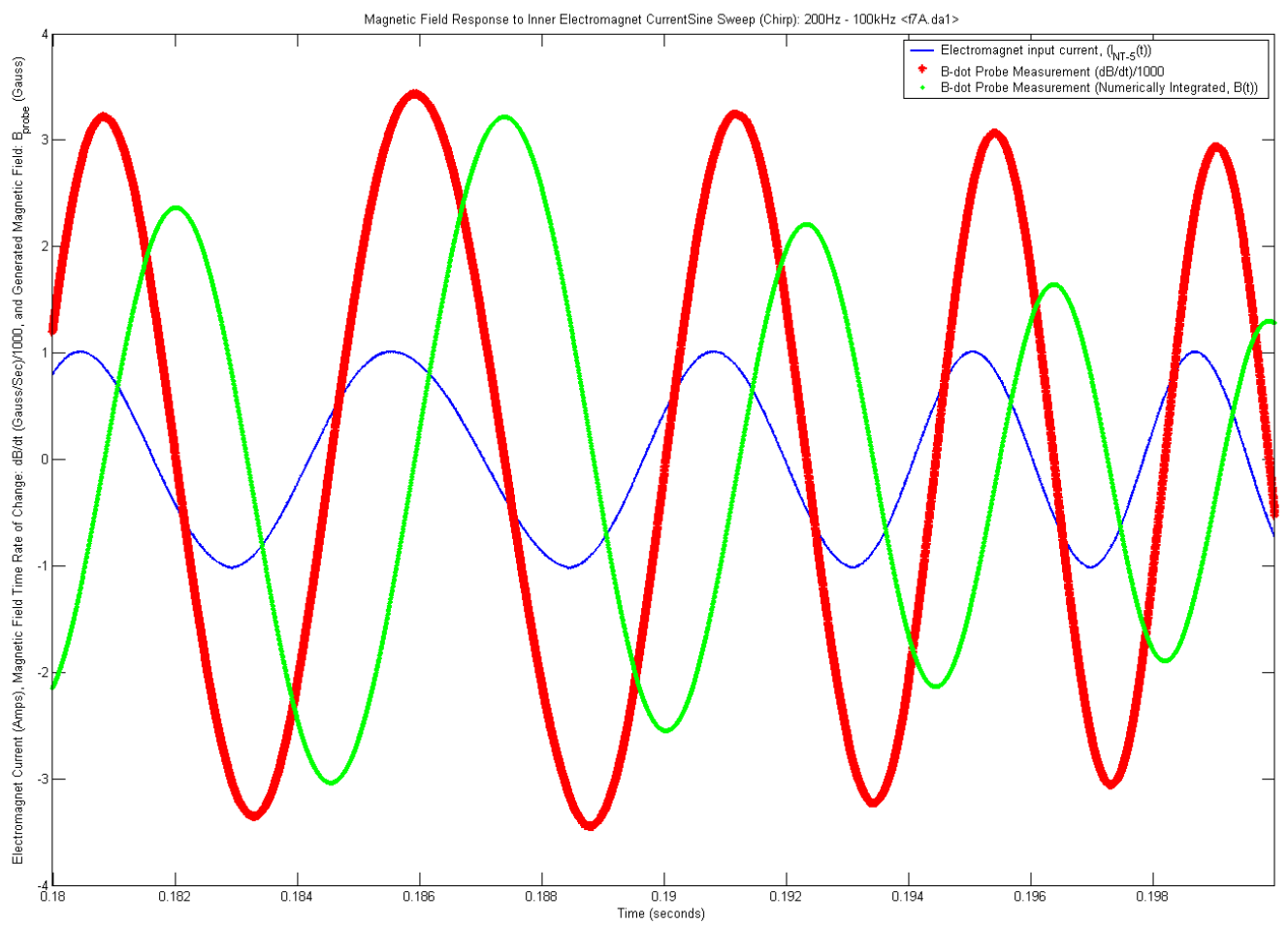

Figure 5. Time response of chirp input signal. Portion of chirp signal inner-magnet input swept from $200 \mathrm{~Hz}$ to $100 \mathrm{kHz}$ over 10 seconds. Except for the numerically integrated signal $\mathrm{B}(\mathrm{t})$, these are the raw signals (unsmoothed) with the appropriate unit conversion. 
In this snapshot the frequency is minimal $(200 \mathrm{~Hz})$ at the second peak and increases as time progresses. Also it can be seen the signal magnitudes for $\mathrm{dB}(\mathrm{t}) / \mathrm{dt}$ and $\mathrm{B}(\mathrm{t})$ decrease with the increasing frequency while the input current signal remains constant. To make this data more useful it is now necessary to study its fft (Fast Fourier Transform).

\section{H. Power Spectrum (FFT)}

The mathematical result of an fft applied to a time domain signal is the set of magnitude (and phase) coefficients of a series of sine waves with linearly varying frequencies (much like a chirp signal) that when added together would recreate the time domain signals. Effectively, the fft transforms the signal from the time domain to the frequency domain. This allows us to study the signals similar to how was shown earlier in figure 4, but in a more efficient manner. With our linear fft the frequency resolution of a single transformation is roughly $0.05 \mathrm{~Hz}$ and plotting all these points (over 5 million) is not trivial; and thus for practical purposes we only plot a fraction of the points but keep in mind that all the points are processed fully prior to plotting. Now to minimize noise in the fft $(0.05 \mathrm{~Hz}$ is a very fine grid) each chirp sweep completes two cycles per 20 second data set and 3 to 6 datasets are collected at each operating condition. Computation of the frequency response of the magnetic field is a linear process and in that sense repeatable (where as the time domain response of a non-triggered chirp is not linear) so that averaging multiple data sets together is quite valid and considerably reduces the scatter of the points in the frequency response plots. For the data in Figure 5 we have applied an fft (and used it along with five other fft's) to produce the following power spectrum plot:

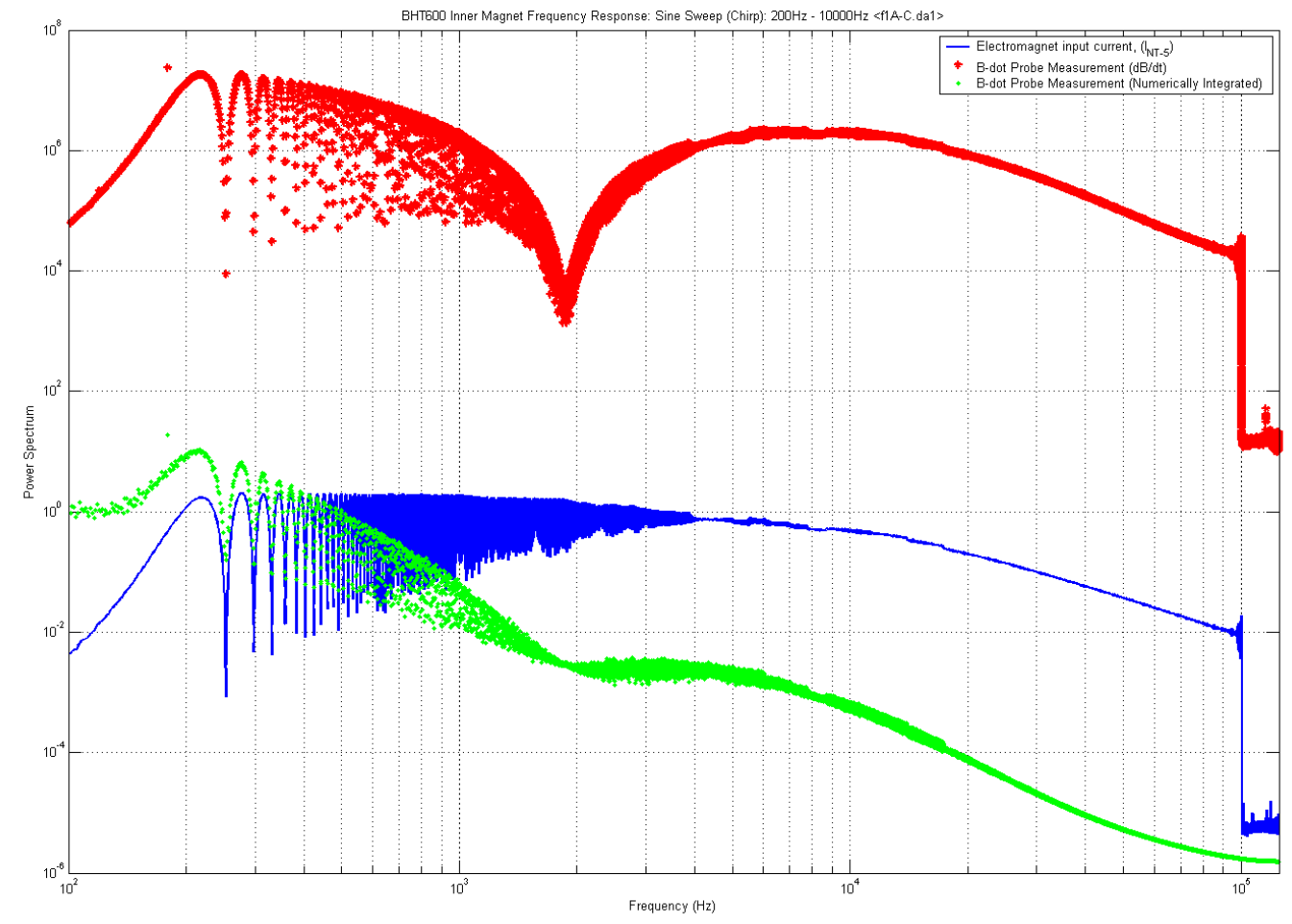

Figure 6. Power Spectrum Response of B-dot probe. Probe location is the center of the discharge channel orientation of probe set to measure radial magnetic flux variations.

The negative spikes on the low frequency side of the data are artifacts from the brief low frequency residence time of the chirp (recall: $10 \mathrm{kHz} / \mathrm{sec}$ is the sweep frequency rate of change). As shall soon be seen, this effect disappears in Bode plot analysis where the artifacts in the input (the solid blue curve in figure 6) exactly cancel the artifacts in the output signals! While the power spectrum if often the main representational use of fft transformed 
data, it is really just the beginning of the analysis from a controller design standpoint. A richer description of the system dynamics of our excited electromagnet is achieved by the use of Bode plot analysis.

\section{Bode Plots Analysis}

A Bode plot is actually two separate plots with identical abscissa (the swept frequencies). The upper of the two plots is similar to the power spectrum plot in that both represent the complex magnitude of the fft terms but for the Bode plot the magnitude is taken from the "transfer function" coefficients (output/input). The transfer function is obtained when the output signal fft (the excited magnetic field) is divided at each frequency by the input signal fft (the excitation electromagnetic current) at each corresponding frequency. Bode plots are incredibly versatile design tools because any two simultaneously acquired signals can be used to create a transfer function relating how the signals correlate to each other. Now, the lower plot in a Bode plot is a representation of the phase difference between the input and output signals $\left(-180^{\circ}\right.$ to $\left.180^{\circ}\right)$. Use of this phase spectrum is critical in stability analysis since an input $180^{\circ}$ off phase with the output may cause it to explode ("destabilize") if the magnitude of the transfer function exceeds a unity threshold. Next we examine the Bode plot obtained from the data in figure 6 by dividing the output signals $(\mathrm{fft}[\mathrm{dB}(\mathrm{t}) / \mathrm{dt}]$ and $\mathrm{ftt}[\mathrm{B}(\mathrm{t})]$ ) and dividing them by the input signal ( $\mathrm{ftt}$ of the electromagnet current):
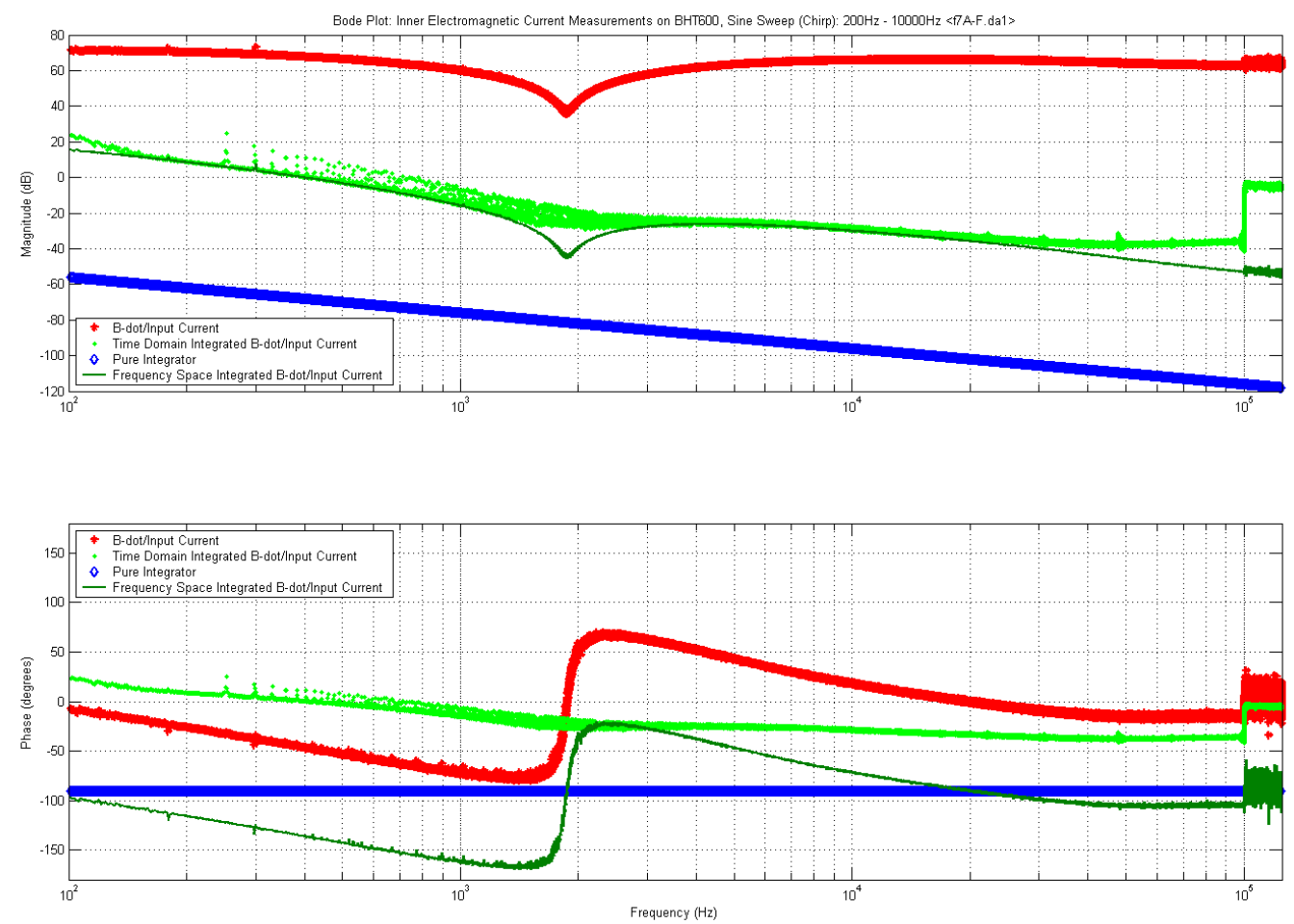

Figure 7. Bode plot for Inner Magnetic Field Frequency Response to Input Current. Chirp input (200Hz$100 \mathrm{kHz}$ ) provided to inner magnet of thruster.

Obtaining the physical magnitude (in gauss units) is not as simple as in the fixed frequency case where Equation 3 was used to directly compute the $B_{\text {rms }}$ values from the $V_{\text {Bdot,rms }}$ values - indeed it doesn't make sense to even compute the $\mathrm{V}_{\text {Bdot,rms }}$ values as the chrip wave is continually changing magnitude and frequency in the time domain. Direct numerical integration of the $V_{\text {Bdot }}(t)$ signal is one approach to obtaining the magnetic field and this has been included in figures 5-7. But the sensitive B-dot probe signal has jitter (variation of the mean) from external sources (radio waves, and other EM radiation from computers and power supplies in the laboratory) that makes the integration prone to cumulative offset errors. For this reason, the signal has been integrated in a second way: by multiplying the transfer function of the $\mathrm{V}_{\text {Bdot }}(\mathrm{t})$ signal by an integrating transfer function. The mathematical Laplace 
transformation of the integration operation is $\mathrm{H}_{\text {integrator }}=1 / \mathrm{z}$, where $\mathrm{z}=-\mathrm{i} * 2 \pi \mathrm{f}$. The complex magnitude and phase of this operator are shown in the preceding Bode plot. Integration of any signal introduces a $-90^{\circ}$ phase shift (at all frequencies) as is clearly seen in the solid blue line in the lower portion of figure 7 . Using this integrator creates the dark green traces in figure 7 which represent the magnitude and phase of the B-dot probe's measurement of the magnetic field relative to the inputted current.

For example, the unity magnetic-field/input-current magnitude point in figure 7 occurs at $400 \mathrm{~Hz}(0 \mathrm{~dB})$. At this frequency the RMS value of the magnetic field (in gauss) will be equivalent the RMS value of the current (in Amps). To check this we have used the LakeShore Gaussmeter to map the frequency dependence of the magnetic field:

\section{Frequency Decay with LakeShore 460 3-Channel Gaussmeter}

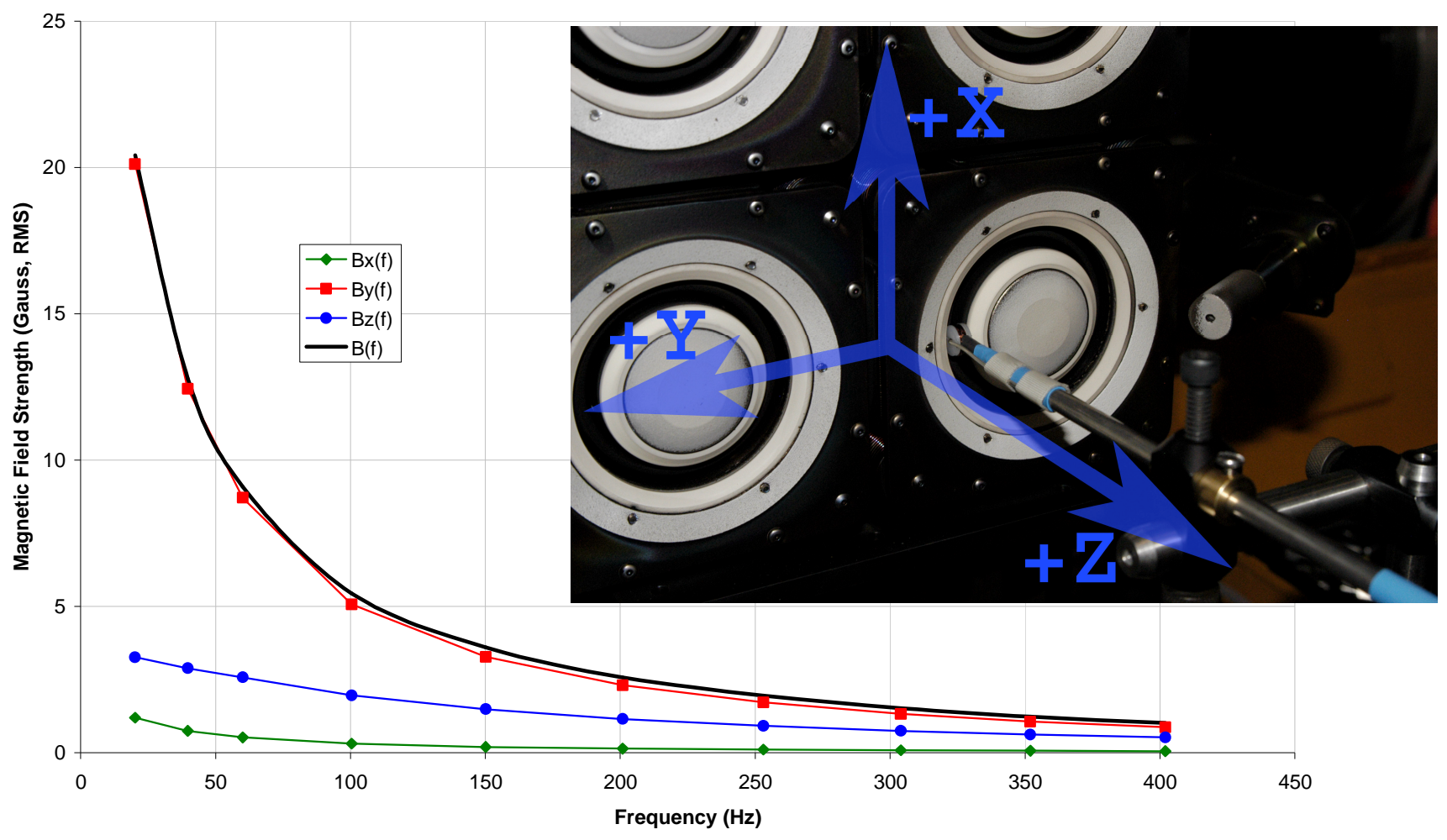

Figure 8. RMS Magnetic Field Measurements with Gaussmeter, all three components of the magnetic field are shown, and an insert photo labels all the coordinates. The probe shown here is actually the B-dot probe but the gauss probe was inserted at the same location to make the RMS field measurements shown. Note: the probe support rods and hardware are all nonmagnetic stainless steel or copper.

Now, on the far right of the above plot, the y-component (shown in red) of the magnetic field measured with the LakeShore Gaussmeter is 0.864 gauss-rms (at $400 \mathrm{~Hz}$ ) while the drive current at this condition was measured as 0.820 Amps! The two values are only $5.1 \%$ off from the expected 1:1 mapping of amps to gauss at this frequency. The phase portion of the Bode plot also indicates that the phase by which the magnetic field lags behind the current is approximately $130^{\circ}$. Now in a pure inductor the current in the electromagnet should lag the voltage driving it by exactly $90^{\circ}$.

Here our treatment of the Hall thruster inner electromagnet as an inductor is not quite ideal. If fact, the Q-factor of our inductor is estimated to be (Reactance/Resistance) $\mathrm{Q}=\mathrm{X} / \mathrm{R}=0.981 \Omega / 1.56 \Omega=0.629$ at $400 \mathrm{~Hz}$. (See Appendix, figure12, for impedance V/I characteristic curve used to compute reactance and estimate inner magnet coil inductance). An ideal inductor would have an infinite Q-factor which shows our IM to be a poor inductor at this frequency! Since the Q-factor varies with frequency we can calculate that even at $100 \mathrm{kHz}$ our Q-factor is 21 which is somewhat closer to a real inductor but not quite ideal. 
With our non-ideal inductor we can still calculate the phase shift between the voltage and current as the arctangent of the Q-factor (neglecting capacitive effects). At $400 \mathrm{~Hz}$ this phase difference is $32.2^{\circ}$ instead of $90^{\circ}$.

\section{J. Bode Plots Analysis Continued: Axial Probe Location}

The Axial position of the B-dot probe was positioned in the center of the Hall thruster discharge channel for the preceding results since the radial magnetic field is maximized at that location. In Hall thruster operation, the bulk of the ionization is believed to occur here and thus it is a sensible location to evaluate the dynamics of the excited magnetic field. Next, a Bode plot is presented that shows how this response varies when moving away from the thruster in the positive $\mathrm{z}$ direction (see figure 8):
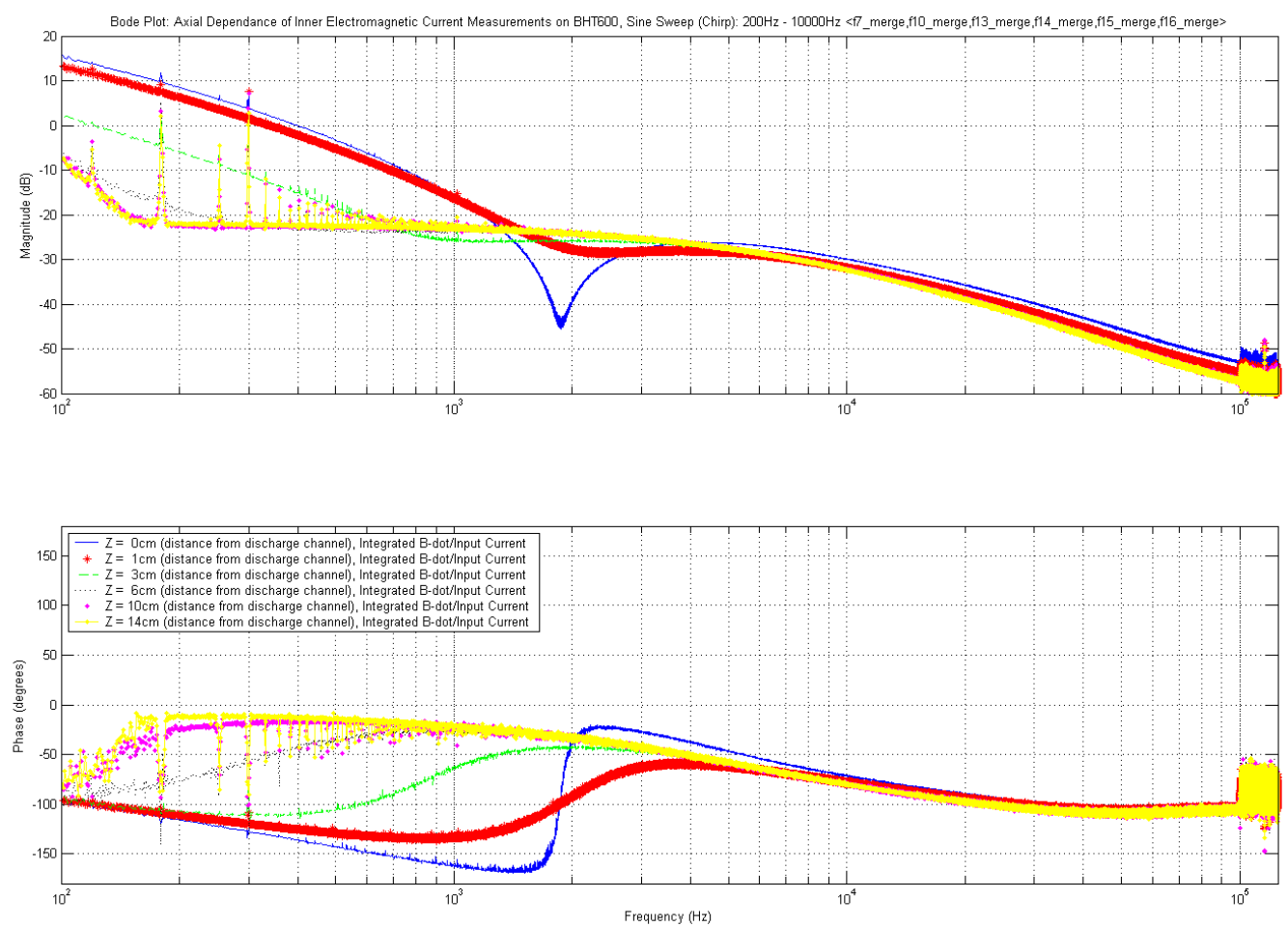

Figure 9. Axial Dependence of Bode plot for Inner Magnetic Field Frequency Response to Input Current. Chirp input $(200 \mathrm{~Hz}-100 \mathrm{kHz})$ provided to inner magnet of thruster.

Here it is seen that the magnetic field drastically loses strength $(-10 \mathrm{~dB})$ at just $3 \mathrm{~cm}$ downstream of the thruster discharge channel. Additionally, the discrepancy between magnetic field strength measured at the six different locations narrows with increasing frequency and by $2 \mathrm{kHz}$ the signals are quite close (within $5 \mathrm{~dB}$ ) to each other (see Appendix, figure 15 for the low frequency field variation at these same locations). This banding together is likely due to the magnetic core absorbing all the magnetic field at these frequencies, but it was a somewhat unexpected result that will be investigated in future experiments.

\section{K. Bode Plots Analysis Continued: Probe Orientation}

Prompted by the unexpected trend observed in figure 9, a set of chirp experiments was performed with the B-dot probe loop rotated to align with the $\mathrm{x}$-coordinate and the $\mathrm{z}$-coordinate (see figure 8) as well as the $\mathrm{y}$-coordinate (or the radial-coordinate) with respect to a single thruster. The following Bode plot shows the magnetic field response curve for these 3 coordinates in a location just $1 \mathrm{~cm}$ axially away from the discharge channel. A fourth curve shows the $\mathrm{z}$-coordinate aligned orientation taken the same distance away axially but aligned with the thruster's center (which would be directly over the top of the inner magnet): 

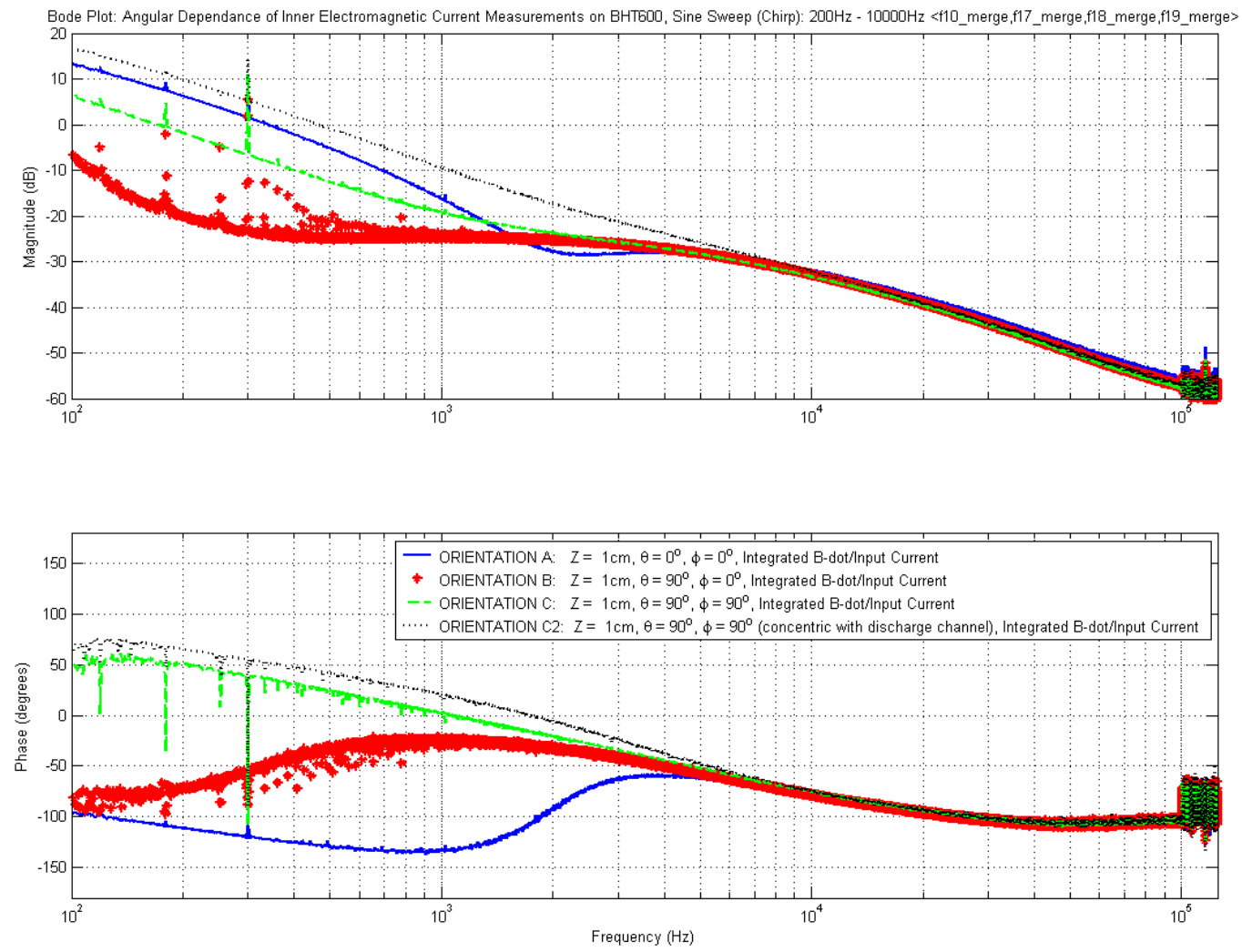

Figure 10. B-dot Probe Orientation Dependence of Bode plot for Inner Magnetic Field Frequency Response to Input Current. Chirp input $(200 \mathrm{~Hz}-100 \mathrm{kHz})$ provided to inner magnet of thruster.

Merging of the signals is again observed at frequencies above $2 \mathrm{kHz}$, and the fourth curve (labeled "Orientation C2") reveals that the magnetic field response is largest in this position and at low frequency $(200 \mathrm{~Hz})$ is nearly equivalent to the response in the middle of the discharge channel (see figure $9, \mathrm{z}=0 \mathrm{~cm}$ trace). But one key distinction between these two cases is the notable linearity of the Orientation C2 trace which lacks the dip that occurs at $1900 \mathrm{~Hz}$ in the magnitude response of the $\mathrm{z}=0 \mathrm{~cm}$ case. For a pure inductor we would expect exactly such a linear trend in magnitude and a constant $90^{\circ}$ phase difference (between the electromagnet voltage and current) which is also observed.

\section{Bode Plots Analysis Continued: Magnetic Field Offset Effects}

The final result presented shows how the inner magnetic field's frequency dependence is affected by additional electromagnet operation (here the orientation and location of the B-dot probe is returned to the nominal configuration as presented in section I.): 

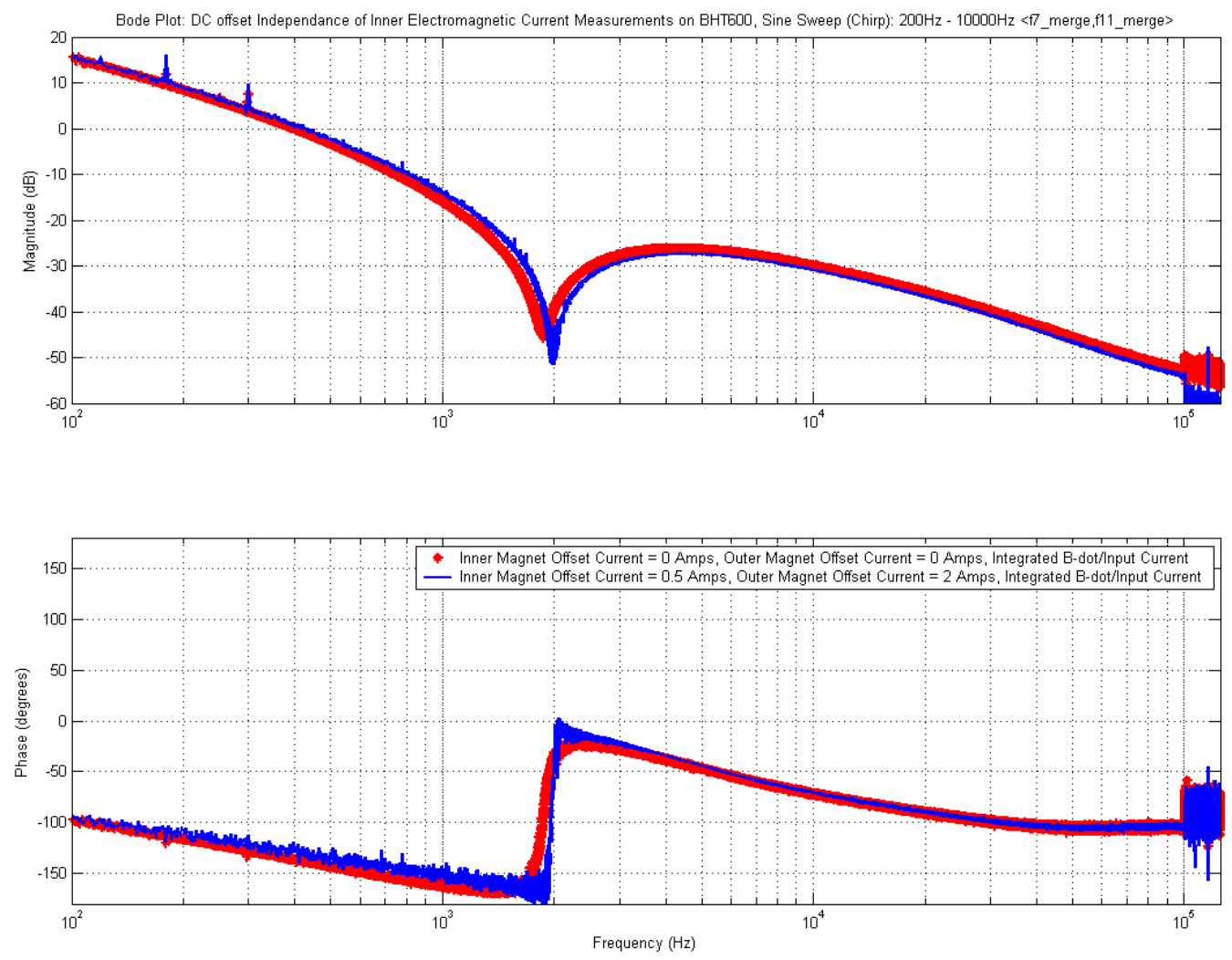

Figure 11. DC Magnetic Field Offset Effect. Adding a constant magnetic field to the chirp experiments performed has little effect of the shapes of the Bode plot response curves in the frequency ranges shown above. Here, a 1Amp peak to peak chirp input $(200 \mathrm{~Hz}-100 \mathrm{kHz})$ is provided to the inner magnet of the thruster with mean value of 0.5 Amps while a DC magnetic field of 2Amps is provided to the outer magnetic coils.

Indeed, adding a DC value to any signal that is applied to a fast Fourier transformation will only alter the fft coefficients at very low (near DC or $0 \mathrm{~Hz}$ ) frequencies. Now, since the output of a B-dot probe is directly proportional to $\mathrm{dB}(\mathrm{t}) / \mathrm{dt}$ we further see that our B-dot probe shouldn't even observe DC magnetic fields as is clear in figure 11.

\section{Conclusion}

The magnetic dynamics of the Busek BHT600 Hall Thruster have been observed in detail by excitation of the electromagnet supply current at frequencies ranging from DC to $100 \mathrm{kHz}$. A Helmholtz coil was constructed calibrated and then used to calibrate the high frequency operation of two B-dot or inductive loop probes. Five different measurements were made of the electromagnet current to ensure accurate magnitude and phase comparisons against the B-dot probe signal. Time domain data was collected and processed into the frequency domain to study power spectrum plots and Bode plots. For the electromagnetic frequency response to the input current, magnitude and phase information was gathered at various axial locations and angular orientations, and under the addition DC magnetic fields. 


\section{Appendix}

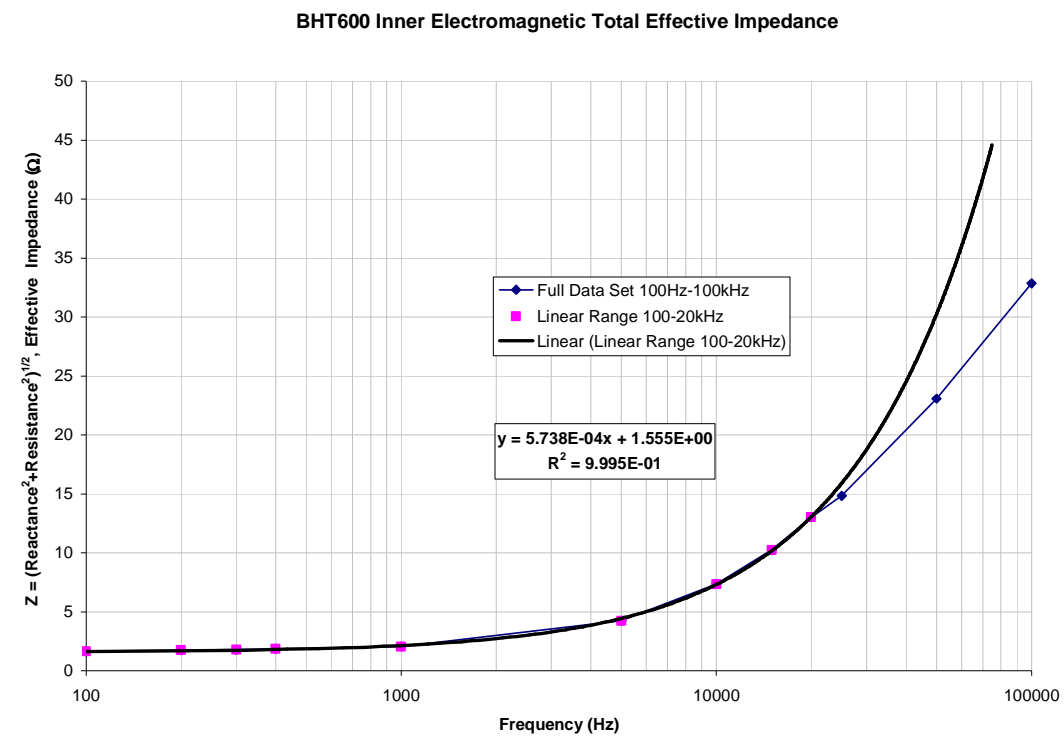

Figure 12. Effective impedance plot. For reactive circuits the concept of resistance is generalized and renamed "Impedance" or Z. Ohm's Law is then generalized to relate complex valued voltages and currents as V=IZ [15]. The above plot uses measured values of voltage and current to calculate the impedance as it varies with frequency. While the $\mathrm{x}$-axis is plotted logarithmically the variation in impedance is quite linear with frequency as the linear fit correlation term, $\mathrm{R}^{2}$, indicates. From this plot the Reactance is determined by using the above y-intercept of $1.56 \Omega$ as the Resistance. The curve that then results is again fitted with a linear curve and the slope is precisely equivalent to the circuit inductance times two pi: $2 \pi \mathrm{L}$. This provides us with an estimate of $99.14 \mu$ Henries as the inductance of the BHT600 inner electromagnetic coil (with core).
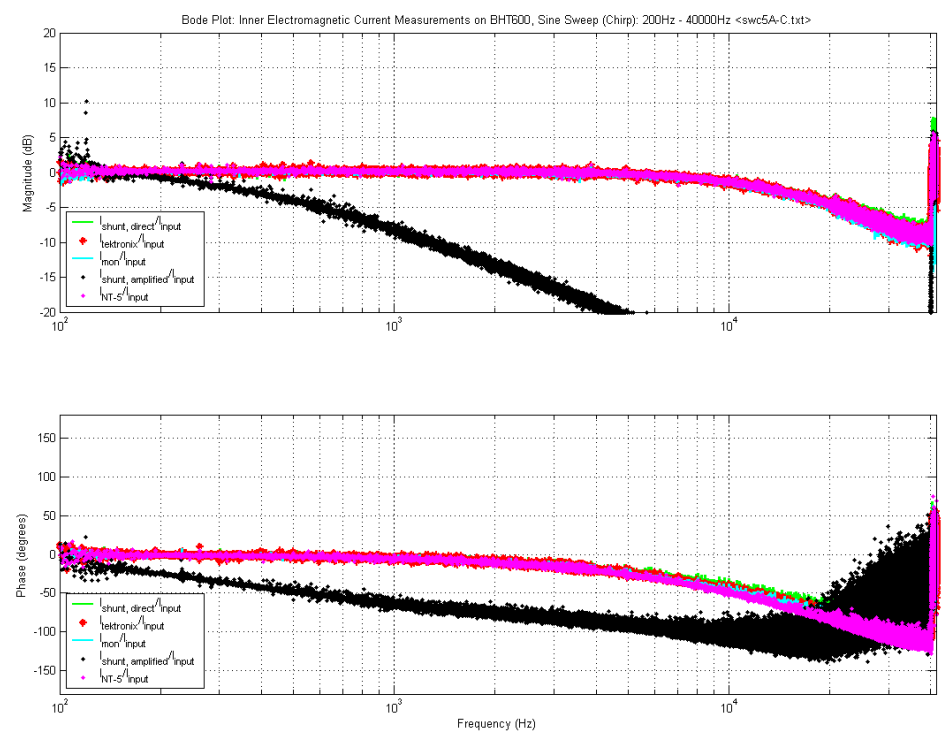

Figure 13. Bode Plot of Power Supply IM3 Current Response from 5 different probes. Chirp excitation frequency ranged from $200 \mathrm{~Hz}-40 \mathrm{kHz}$. The odd decay of the amplified (and voltage isolated) shunt measurement in this preliminary test is likely due to a flaw in the circuit since the expected frequency response shouldn't drop by more than $5 \mathrm{~dB}$ until $20 \mathrm{kHz}$ (the power supply's manufacturer's specified freq. response). The amplified shunt data was neglected upon this observation. 

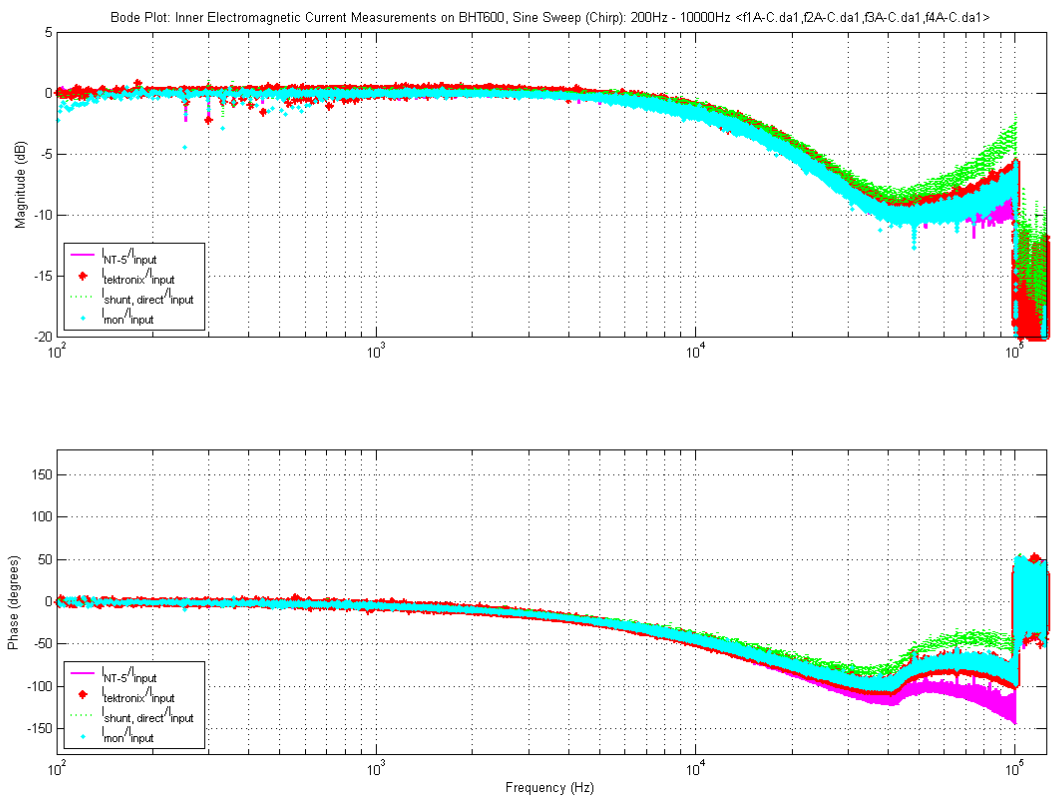

Figure 14. Bode Plot of Power Supply IM3 Current Response from 4 different probes. This is similar to the previous figure 13, except that the chirp excitation frequency range increased to $200 \mathrm{~Hz}-100 \mathrm{kHz}$. The increase in the measured current response at higher frequencies $(40 \mathrm{kHz}-100 \mathrm{kHz})$ is unusual but likely is due to the inner electromagnetic coil becoming a more pure inductor (Q-factor increases with frequency to $21 \mathrm{at} 100 \mathrm{kHz}$ ).

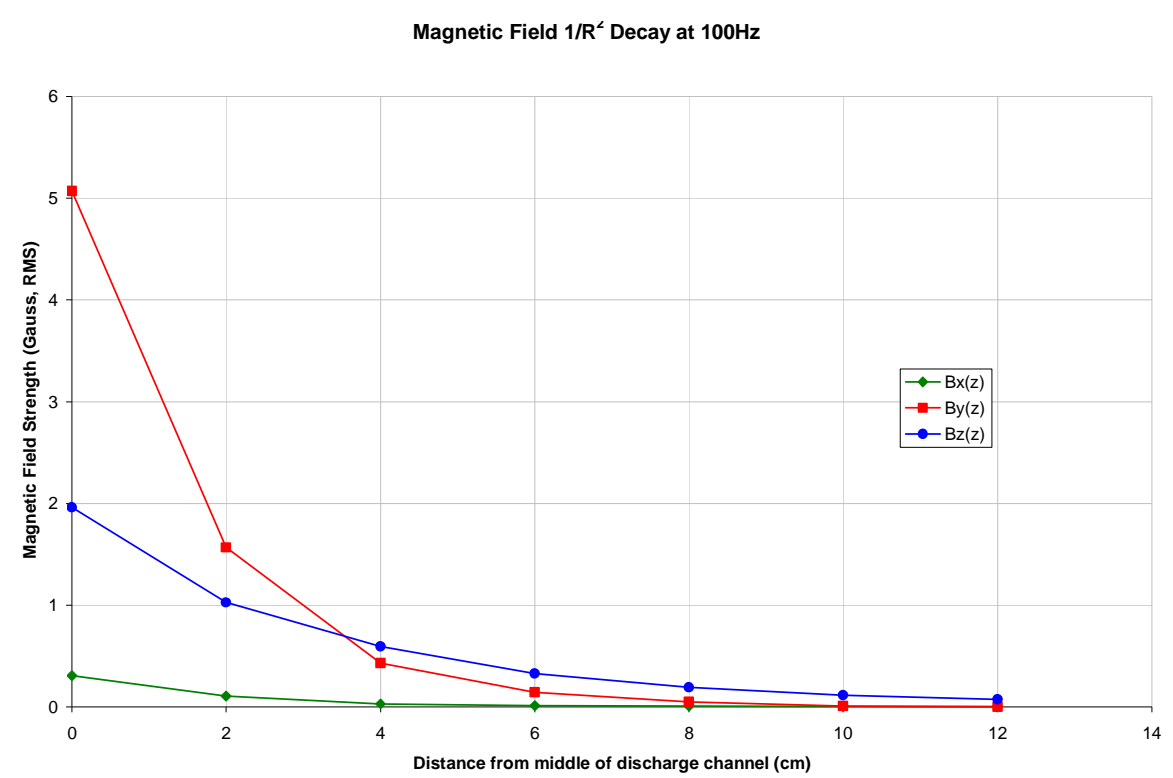

Figure 15. Axial Dependence of low frequency Magnetic Field. Using the LakeShore 3-Channel Gaussmeter, the decrease in magnetic field is observed to follow the expected $1 / \mathrm{R}^{2}$ law for excitation at a fixed frequency of $100 \mathrm{~Hz}$. 
BHT600 Hall Thruster Inner Magnet Frequency Dependance

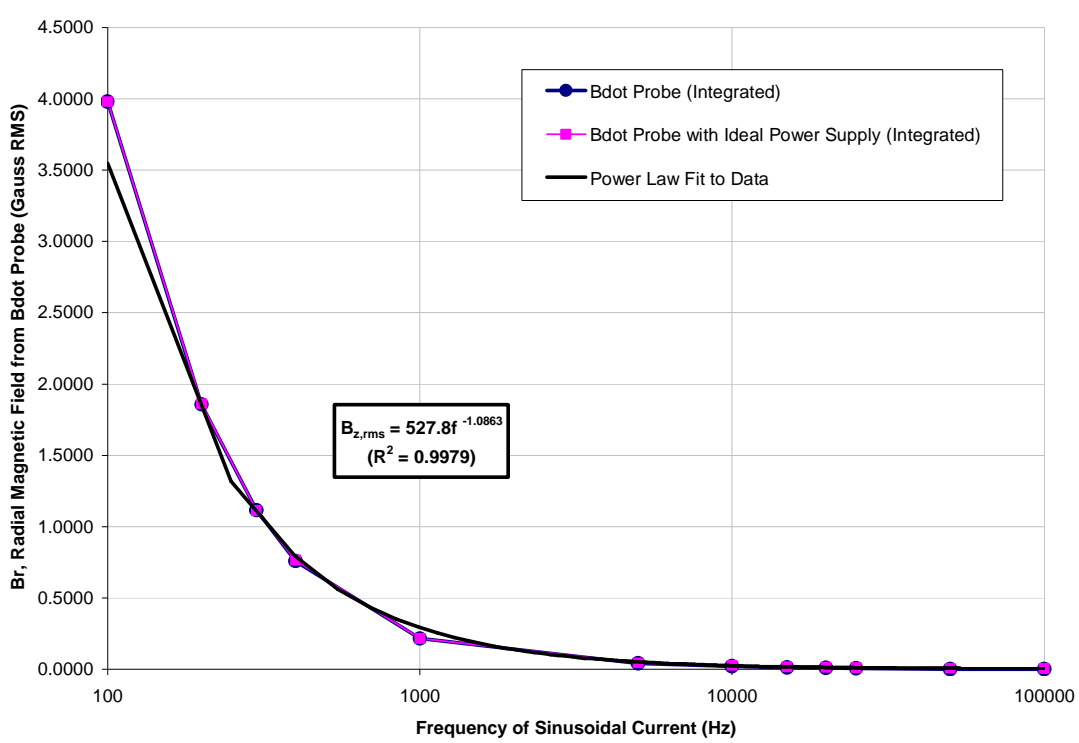

Figure 16. Fixed Frequency Measurements of Magnetic Field using B-dot Probe. This plot is rather similar to figure 7 but the nice power-law fit to the data is an interesting result. Additionally, in this plot the magnitude decay of the power supply is artificially compensated for (magenta squares) and this set of compensation factors is applied to the integrated (as per equation 3) B-dot field measurements to show that even a perfect power supply would produce a magnetic response with the same sharp response decay seen in the obtained power-law.

\section{References}

1. Tajmar, M., Advanced Space Propulsion Systems. 2003, Austria: Springer. 80-92.

2. Beal, B.E., Clustering of Hall Effect Thrusters for High-Power Electric Propulsion Applications. Ph.D. Dissertation, University of Michigan, 2004.

3. Choueiri, E.Y., Plasma oscillations in Hall thrusters. Physics of Plasmas, 2001. Vol. 8, No. 4.

4. Choueiri, E.Y., Characterization of Oscillations in Closed Drift Thrusters. 30th AIAA Joint Propulsion Conference and Exhibit, 1994. AIAA-94-3013.

5. Chesta, E., et al., A Characterization of Plasma Fluctuations within a Hall Discharge. IEEE Transactions on Plasma Science, 2001. Vol. 29, No. 4.

6. Gascon, N., et al., Signal Processing and Non-Linear Behavior of a Stationary Plasma Thruster: First Results. 35th AIAA Joint Propulsion Conference and Exhibit, Los Angeles, CA., 2000. AIAA-99-2427.

7. Litvak, A.A., Y. Raitses, and N.J. Fisch, Experimental studies of high frequency azimuthal waves in Hall thrusters. Physics of Plasmas, 2004. Vol. 11, No. 4.

8. Chesta, E., B. Meezan, and M.A. Cappelli, Stability of a magnetized Hall plasma discharge. Journal of Applied Physics, 2001. Vol. 89, No. 6.

9. Baranov, V.I., et al., Theory of oscillations and conductivity for Hall thruster. $32^{\text {nd }}$ AIAA Joint Propulsion Conference and Exhibit, Buena Vista, FL, 1996. AIAA-96-3192.

10. Lobbia, R.B. and A.D. Gallimore, Evaluation and Active Control of Clustered Hall Thruster Discharge Oscillations. 41st AIAA Joint Propulsion Conference and Exhibit, Tucson, Arizona, 2005. AIAA-2005-3679.

11. Kang, B.K. and M.A. Lieberman, Axial Feedback Stabilization of Flute Modes for Mirror Machines. IEEE Transactions on Plasma Science, 1988. Vol. 16 No. 1: p. p. 27-38.

12. Lao, L.L., et al., Physics and control of ELMing H-mode negative-central-shear advanced tokamak ITER scenario based on experimental profiles from DIII-D. Nuclear Fusion, 2003. 43: p. p.1023-1030.

13. Peterson, P.Y., A.D. Gallimore, and J.M. Haas, Experimental Investigation of a Hall Thruster Internal Magnetic Field Topography. 27th International Electric Propulsion Conference, Pasadena, CA, 2001.

14. Moore, J.H., C.C. Davis, and M.A. Coplan, Building Scientific Apparatus. 3rd ed. 2003, Massachusetts: Perseus Books.

15. Horowitz, P. and W. Hill, The Art of Electronics. 2 ed. 2001, Massachusetts: Cambridge Univeristy Press. 